\title{
Low-fat, lactose-free and leucine-enriched chocolate cow milk prototype: A preliminary study on sensorial acceptability and gastrointestinal complaints following exhaustive exercise
}

Cristiano D. da Silva ${ }^{1,2^{*}}$ D, Dirce R. de Oliveira ${ }^{3}$, Ítalo T. Perrone ${ }^{4,5}$, Carlos H. Fonseca ${ }^{6}$ and Emerson S. Garcia ${ }^{7}$

\begin{abstract}
Background: Chocolate milk has gained recent scientific support as a recovery drink. However, it is known that high exercise-demand triggers gastrointestinal discomfort which continues post-exercise, thereby hindering this nutritional strategy. In addition, those who are lactose intolerant cannot benefit from a milk-based beverage. Thus, the aim of this preliminary study was to develop a low-fat, lactose-free, and leucine-enriched chocolate cow milk prototype $(\mathrm{CML})$ representing nutrition-related recommendations for football players, as well as assess athletes' individual subjective outcomes for gastrointestinal complaints and sensorial acceptability in a field-based setting following strenuous team-sport physical demands.

Methods: This study followed a single group and repeated-measured design with 10 football players ( $23 \pm 2 \mathrm{yrs}$., $74 \pm 14 \mathrm{~kg}, 174 \pm 5 \mathrm{~cm}$ ) who consumed CML following a 90-min football match simulation protocol (FMP). The total CML intake to achieve $0.150 \mathrm{~g}$ leucine. $\mathrm{kg}$ [BW]. $\mathrm{h}^{-1}$ occurred in aliquots of 50,30 and $20 \%$ at $0-, 45$ - and 75 -min post-FMP, respectively. Athletes were evaluated by the prevalence, the type and severity (bloating, nausea, flatulence, and gastric reflux) of gastrointestinal complaints and sensorial acceptability (overall perception, appearance, consistency, and flavour) after drinking each aliquot in a 4-h recovery period.

(Continued on next page)
\end{abstract}

\footnotetext{
* Correspondence: cristianodiniz.silva@gmail.com

1Department of Physical Education, Institute of Life Sciences, Federal

University of Juiz de Fora, Campus: Governador Valadares, Rua Manoel Byrro,

241 - Vila Bretas, Governador Valadares, MG 35010-260, Brazil

${ }^{2}$ School of Physical Education, Physiotherapy and Occupational Therapy,

Federal University of Minas Gerais, Belo Horizonte, MG, Brazil

Full list of author information is available at the end of the article
}

C C The Author(s). 2021 Open Access This article is licensed under a Creative Commons Attribution 4.0 International License, which permits use, sharing, adaptation, distribution and reproduction in any medium or format, as long as you give appropriate credit to the original author(s) and the source, provide a link to the Creative Commons licence, and indicate if changes were made. The images or other third party material in this article are included in the article's Creative Commons licence, unless indicated otherwise in a credit line to the material. If material is not included in the article's Creative Commons licence and your intended use is not permitted by statutory regulation or exceeds the permitted use, you will need to obtain permission directly from the copyright holder. To view a copy of this licence, visit http://creativecommons.org/licenses/by/4.0/. The Creative Commons Public Domain Dedication waiver (http://creativecommons.org/publicdomain/zero/1.0/) applies to the data made available in this article, unless otherwise stated in a credit line to the data. 
(Continued from previous page)

Results: The CML showed higher scores for "Product Acceptability Index" (88\%) and sensorial acceptability ( 8 in 9 -point hedonic scale). Kendall's W with bootstrapped resample $(95 \% \mathrm{Cl})$ revealed agreement among respondents as "moderate" (overall perception, flavour) to "strong" (appearance, consistency) and with no significant agreement differences between rater response in the timeline analysis (0.57 up to 0.87; $p>0.05$ ). Agresti-Caffo add-4 analysis (95\% confidence interval, [95\% Cl]) revealed no differences in each time-point analysis versus baseline for athletes classified as having severe gastrointestinal symptoms, but confirmed concern with bloating (three athletes showed a transient response at 2-h and only one continued until 3-h; $p=0.051$ ).

Conclusions: These preliminary findings suggest that CML presents good taste and high acceptability by the sampled athletes. Thus, CML may be an alternative sport drink for immediate post-workout supplementation to overcome the energy deficit, offer co-ingested leucine, maintain palatability and adherence including lactose intolerance following a team sport-specific fatigue.

Trial registration: $\mathrm{RBR}-2 \mathrm{vmpz}, 10 / 12 / 2019$, retrospectively registered.

Keywords: Soccer player, Athlete, Discomfort, Sports nutrition, Product acceptability, Workout recovery, Milk-based beverage

\section{Introduction}

Nutrition is considered one of sports performance pillars, and fulfilling the post-exercise nutrition-related recommendations is fundamental for the recuperative and adaptive process effectiveness. Hence, an effective recovery strategy between workouts or inside competitive events may maximize an adaptive response to a variety of fatigue-mechanisms improving muscle structure and functionality and thereby increasing exercise tolerance. In addition to enabling better adaptability conditions, an effective recovery intervention can also gradually make an athlete's body become better for particular features of sport demands which are important to performance and injury prevention. Thus, an effective nutritional recovery intervention taking into account the food supply timing and quality is considered fundamental [1,2].

Current post-workout nutrition-related recommendations for multiple sprint sports athletes point to the offer of a carbohydrate-protein mixture (CHO:PRO) [2-4]. The need for this CHO:PRO combined intervention is justified by previous evidence regarding an increased muscle glycogen storage, muscle damage amelioration, and greater acute and chronic training adaptations in the above mentioned sport-workload context [2]. By addressing such mechanisms in detail, it is evident that the CHO:PRO mixture improves the acute recovery process via stimulation of muscle protein synthesis (MPS), as well as the activation of the target mechanism of rapamycin (mTOR), signalling a pathway $[5,6]$ and a more effective glycogen storage via insulinotropic response $[7,8]$.

Considering CHO:PRO natural mixtures and other potential nutrients, milk and chocolate milk have recently gained scientific support as recovery drinks in adults [911] and adolescent athletes [12]. Despite the promising aspect of chocolate milk as a recovery drink, those lactose intolerants will not be able to benefit from this strategy. In addition, athletes may experience gastrointestinal discomfort and reduced appetite after highdemand sports activities [13, 14]. In this sense, there is still a lack of evidence regarding the use of chocolate milk beverages and prevalence of gastrointestinal discomfort, observing conflicting results $[10,15]$. Furthermore, chocolate milk may be a high palatability supplement, but few studies have tested its sensorial acceptability [16].

Exercise scientists, support professionals, and athletes may have a strong interest in special formulation alternatives to conventional sports drinks, such as chocolate milk, which are designed to meet a high metabolic cost in exhaustive team sports. Another reason for the interest is to find an alternative beverage proposal which does not compromise adherence to the continuity recovery diet plan due to gastrointestinal distress as demonstrated in commercial recovery aid [16] or because high rate of diuresis impairing rehydration as occurred in postworkout plans using conventional sports drinks [11, 13]. In this context, we developed a low-fat, lactose-free, and leucine-enriched chocolate cow milk prototype (CML). With regard to this unprecedented experimental sportspecific beverage, the aim of this preliminary study was to propose an empirical clarification producing the CML, testing its supplementation use and analysing athletes' individual subjective outcomes on gastrointestinal complaints and sensorial acceptability. In the trial, we proposed a practical ("ready-to-drink") and functional intake protocol (lactose-free and representing nutritionrelated recommendations for football players), and maintained similar conditions to real-life use following a football-specific acute fatigue. 


\section{Methods}

\section{Experimental design}

This study was preliminary and focused on individual subjective outcomes, and therefore it provides guidelines to support a large-scale randomized controlled trial in the future. Moreover, a real consumer's emotional state (i.e. post-exhaustive exercises) was considered appropriate to simulate a real beverage consumption, constituting a context which could compromise the acceptance and sensory rejection of the product [17]. Therefore, a repeated measures independent group was adopted, and a randomized or placebo-controlled trial was not considered adequate in this preliminary study. As an attempt to make the study more controllable, athletes did not concomitantly intake water or other foods with CML consumption, avoiding apparent bias in subjective outcomes, which are the focus of this study. Researchers were not involved in participant recruitment or any assessments.

In the experimental phase, the study began with a particular CML proposition and process (see the beverage composition and processing proposal below). Athletes had to report to the laboratory on two different occasions. The first visit preceded the main trial to sample descriptive characterization, individual habitual food intake data for prescribing a pre-trial meal and beverage intake (e.g., leucine intake and ingestion volume), and conduct trial familiarization with a football-match simulation protocol. The second visit involved a pre-trial meal and standardized hydration; baseline data; fatiguing exercise via a standardized football match-related protocol; CML ingestion; and post-intake data in the recovery period timeline. The outcome measures of interest were gastrointestinal complaints (7 within-effect measurement times) and taste (3 within-effect measurement times) within a $4 \mathrm{~h}$ follow-up period.

\section{Participants}

Ten male ( $23 \pm 2$ yrs., $74 \pm 14 \mathrm{~kg}, 174 \pm 5 \mathrm{~cm}$ ) universitystandard football outfield players (defenders $[N=3]$; midfielders $[N=5]$; attackers $[N=2]$ ) were recruited from a randomization procedure in the team squad to participate in this study. The subjects were informed of the purpose, procedures, and associated risks prior to signing an informed written consent obtained according to a protocol approved by the Research Ethics Committee of Federal University of Minas Gerais, Brazil (CAAE: 32153514.5.0000.5149). Subjects finished their competitive season (of the University Athletic League) approximately 2 weeks before the study and were engaged in unstructured training to keep fit. Players trained 4-5 days per week on the field (technical-tactical) during the season, including 2 conditioning-based sessions (i.e., high-intensity training, sprinting, strength/plyometric work) and one match. Health and well-being were prioritized, thus the sample size and all subjects in this preliminary study were not lactose intolerant, nor allergic to dairy products, with these same subjects being interviewed in the sample selection stage.

\section{Beverage composition and processing}

The CML prototype was prepared approximately 1 week before consumption using a cow milk and dairy product platform for mixing the ingredients until homogeneous and then pasteurization. Once ready, the CML was kept at refrigerator temperature and tested according to Brazilian food regulations and microbiological conditions for milk and dairy products - IN 16/2005 [18]. Food microbiology analyses were performed using Petrifilm 3 $\mathrm{M}^{\mathrm{m}}$ methodologies (3 M Company, St. Paul/MN, USA), in agreement with standards of Brazilian specific legislation - RDC 12/2001 [19].

In the sport-specific beverage formulation, the first order was to consider the $\sim 3: 1 \mathrm{CHO}: \mathrm{PRO}$ ratio (nutritional content per $200 \mathrm{~mL}=204 \mathrm{kcal} ; 35 \mathrm{~g}$ [CHO]; $12 \mathrm{~g}$ [PRO] and $3.3 \mathrm{~g}$ [FAT]) to simulate chocolate milks presented in previous studies [9-11] and nutrition-related recommendations $[2,20]$ to formulate a more effective recovery drink to be used immediately after game or trainings in football players. In addition, functional enrichment in the beverage was thought to improve its amino acid profile. Thus, the CML would enable synergistic effects in the recovery process or physical stress adaptation, evidenced by the use of protein mixture, leucine hydrolysates, and $\mathrm{CHO}$ in insulin response amplification [21-24]. Although naturally present in cow's milk, essential amino acids (EAAs) are critically needed for achieving maximal rates of MPS, requiring high quality protein sources (which contain EAAs, including leucine) [20]. Therefore, the CML and its use were designed to offer a continuous supply of amino acids in circulation, suggested at the design conception time to be at a leucine intake of $\sim 0.150$ g.kg. [BW] $]^{-1}$ or $\sim 10$ g.h $[B W]^{-1}[22,23,25]$ and $6-20 \mathrm{~g}$ of EAA $[24,26]$. Leucine intake of CML was approximately $70 \%$ below the tolerable upper limit $[27,28]$.

\section{Procedures}

In the first visit, the athletes were characterized (weight and height) and familiarized using a standardized 90min football-match simulation protocol (FMP) [29, 30]. The energetic value of the athletes' habitual intake was determined by registering their usual food diet during three consecutive days, and also including their average weekend consumption [31]. We calculated their habitual macronutrient consumption using a nutritional software analysis (Avanutri ${ }^{\oplus}$, Rio de Janeiro, Brazil). At the end of the first visit procedures, they were asked to abstain 
from alcohol consumption and to moderate strenuous activity $24 \mathrm{~h}$ in advance, as well as caffeine-containing products for $12 \mathrm{~h}$ prior to the trial. The athletes were required to keep a 3-day written record of their habitual food intake.

On the second visit, the athletes arrived at the laboratory in the morning following an overnight fast $(8 \mathrm{~h})$. A urine sample was collected from each athlete to measure and compare their isovolumetric water quantities before and during FMP. Then, all athletes received a pre-trial standardized meal $(\sim 380 \mathrm{kcal}, 68 \mathrm{~g}$ of $\mathrm{CHO}, 11 \mathrm{~g}$ of PRO, $7 \mathrm{~g}$ of FAT) and $5 \mathrm{~mL} . \mathrm{kg}[\mathrm{BW}]^{-1}$ of water [32]. Athletes classified into hypohydrate state (Urine Specific Gravity; USG $\geq 1.020$ a.u.) [33] received an additional 2 mL.kg $[\mathrm{BW}]^{-1}$ of water [34]. After the meal, the athletes rested for $75 \mathrm{~min}$ and were then invited to wear the appropriate clothing and footwear to perform $15 \mathrm{~min}$ of standard football match-day warm-up and stretching. The hydration in FMP occurred with water $(2 \mathrm{~mL} . \mathrm{kg}$ $[\mathrm{BW}]^{-1}$ ) every $15 \mathrm{~min}$ up to $75 \mathrm{~min}$ to ensure adequate hydration [32]. FMP was a 90-min fatiguing exercise protocol $\left(\mathrm{T}-\mathrm{SAFT}^{90}\right.$ ) corresponding to an official football match played from audio commands [29, 30]. Each player used a strap equipment component of Polar Team System $^{\circ}$ (Polar Electro Oy, Kempele, Finland). FMP was conducted on a natural grass football pitch at $25 \pm 1{ }^{\circ} \mathrm{C}$ and $63 \pm 1 \% \mathrm{RH}$. The athletes were referred for CML supplementation and passive recovery at the end of the FMP. The athletes were placed in thermoneutral room $\left(23 \pm 1{ }^{\circ} \mathrm{C}, 60 \pm 1 \mathrm{RH}\right)$ during and after the pre-FMP meal, as well during the treatment and recovery period. A scheme of the study protocol timeline and collection plan of each variable is shown in Fig. 1.

\section{CML treatment and recovery period}

The athletes remained in the laboratory for $4 \mathrm{~h}$ of relaxed recovery following the FMP, where they received the total volume of CML for treatment $(\sim 630 \mathrm{~mL})$ in aliquots at three separate moments: $50 \%$ at time $0,30 \%$ at $45 \mathrm{~min}$ and $20 \%$ at $75 \mathrm{~min}$. This hypothetical-deductive CML intake plan aimed to increase adherence to treatment (appetite appeal and less satiety, and so, ingest the final treatment volume), and especially to allow a continuous supply of circulating amino acids and insulinemic response. Athletes were seated and instructed to drink the entire offered volume quota in $5 \mathrm{~min}$. A lab technician was responsible for the timing, scheduling, and delivery of beverage. The beverage was served at $4{ }^{\circ} \mathrm{C}$.

\section{Subjective measures}

A questionnaire was conducted in pre-trial (baseline), immediately post-FMP and every hour up to 4-h postintake for evaluating the type and severity of gastrointestinal symptoms (bloating, nausea, flatulence, and gastric reflux) on a scale of "1" to "10" arbitrary unit (a.u.), with " 1 " referring to "no symptoms" and " 10 " to "severe symptoms" [35].

Sensory acceptability was evaluated in the following attributes: overall perception, appearance, consistency, and

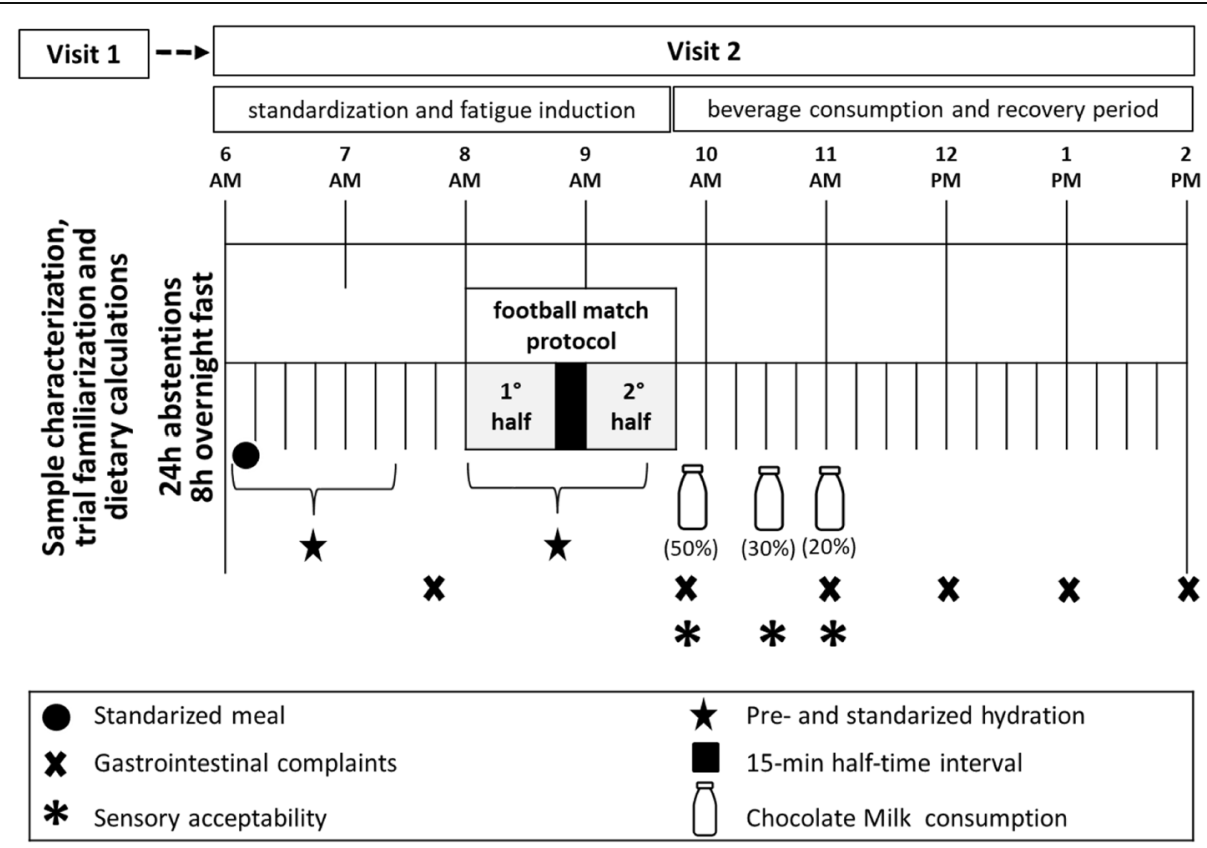

Fig. 1 Schematic diagram for the study protocol timeline including visit requirements, fatigue induction via a standardized 90-min football match simulation protocol (FMP), measurements flow, treatment with experimental chocolate cow milk beverages and recovery period 
flavour. Athletes were requested to rate the drink according to a 9-point hedonic scale from "1" ("I did not like it very much") to "9" ("I really liked it") [36]. The equation $[\mathrm{PAI} \%=$ average grade obtained for the product * 100/maximum grade given to the product] was adopted to calculate the "Product Acceptability Index" (PAI) [37].

\section{Data analysis}

Sample description and intake volume were presented with means \pm sd. Gastrointestinal complaint severity were divided into two categories: severe symptoms and less severe symptoms [35]. Prevalence of severe symptoms were only registered when a score of " 5 " or higher out of "10" a.u. was given. Less severe symptoms were registered when a score below " 5 " was given. Taste and overall perception were presented as frequency of each sensory attribute. Friedman rank sum test was used to verify the sensorial acceptability time effect. Comparisons of proportions of athletes with severe gastrointestinal symptoms in each time point versus baseline was calculated using the multivariate normal distribution (Dunnett-adjusted). Standard normal quantiles and estimates with $95 \%$ confidence interval $(95 \% \mathrm{CI})$ were calculated by the Agresti-Caffo add-4 [38]. The degree of sensorial acceptability agreement between responders from each aliquot intake was analysed using Kendall's W coefficients agreement degree $(0 \leq \mathrm{W} \leq 1,1$ representing perfect concordance) with bootstrapped reanalysis (95\% CI; creating 1000 bootstrap from the original data). All statistical analyses were performed using $\mathrm{R}$ software environment for statistical computing ( $\mathrm{R}$ Core Team, version 3.6.0). The statistical significance was set at $p<0.05$ for all analyses.

\section{Results}

The metabolic load impact variables of the FMP were: exercise intensity $\left(85 \pm 7 \% \mathrm{HR}_{\text {peak }}\right)$, body mass change ($2.3 \pm 0.9 \%)$ and USG (1018 \pm 6 a.u.). According to the subjects' characteristics $(23 \pm 2$ yrs., $74 \pm 14 \mathrm{~kg}, 174 \pm 5$ $\mathrm{cm})$ and individual desired dose of leucine ingestion, the CML average intake was $625 \pm 61 \mathrm{~mL}(312 \pm 31 \mathrm{~mL}$ at time $0 ; 187 \pm 18 \mathrm{~mL}$ at $45 \mathrm{~min}$ and $125 \pm 12 \mathrm{~mL}$ at 75 min post-FMP, respectively). The total caloric intake average was $637 \pm 62 \mathrm{kcal}$.

Most athletes (70\%) did not report any severe gastrointestinal symptoms in 4-h of treatment and recovery period (Table 1). However, a transient effect was observed 2-h post-intake on three athletes $(30 \%)$ who reported severe scores for bloating. Two of these three previously mentioned athletes presented concomitant nausea and gastric reflux, and the one other volunteer presented flatulence. Comparisons of proportions of athletes with severe gastrointestinal symptoms revealed no differences in each time-point analysis versus baseline, but confirmed concern with bloating symptom (AgrestiCoull add-4 analysis $[-0.26,0.43,95 \% \mathrm{CI}] ; p=0.051$; Table 1). Only one volunteer who presented "bloating" had a follow-up of this symptom 3-h post-intake. No participant abandoned this study due to gastrointestinal distress.

Quantitative analyses via the Friedman test showed that there was no significant difference for the aliquot time effect for all of the attributes' acceptance $(p>0.05)$. The qualitative results of the sensory acceptability for CML revealed that most athletes $(60-80 \%)$ answered as "I really liked it" and "really enjoyed it" (Table 2), with the degree of agreement among responders observed as "moderate" (overall perception, flavour) to "strong" (appearance, consistency), and with no significant agreement differences between the raters' responses in the different evaluation times (Kendall's W agreement degree: 0.57 up to $0.87 ; p>0.05$ ).

The overall sensorial evaluation showed higher scores ( 8 out of 9-point) for all attributes' acceptance criterion (e.g., perception, appearance, consistency, and flavour; Table 3). The CML had a high classification score regarding the PAI (88\%).

\section{Discussion}

Post-workout supplementation strategies are an interesting topic for athletes and well-trained people. Therefore, it is necessary to formulate and test new sports drinks with compositions for particular workload recovery

Table 1 Prevalence of severe gastrointestinal complaints during experimental chocolate cow's milk supplementation

\begin{tabular}{lllllll}
\hline Complaint & post-FMP & 1-h post-intake & 2-h post-intake & 3-h post-intake & 4-h post-intake & $\boldsymbol{p}$-value \\
\hline bloating & $0[0.0 ;-0.29,0.29]$ & $0[0.0 ;-0.29,0.29]$ & $3(30 \%)[0.3 ;-0.16,0.66]$ & $1(10 \%)^{b}[0.1 ;-0.26,0.43]$ & $0[0.0 ;-0.29,0.29]$ & 0.051 \\
nausea & $0[0.0 ;-0.29,0.29]$ & $0[0.0 ;-0.29,0.29]$ & $2(20 \%)^{a}[0.2 ;-0.21,0.55]$ & $0[0.0 ;-0.29,0.29]$ & $0[0.0 ;-0.29,0.29]$ & 0.127 \\
flatulence & $0[0.0 ;-0.29,0.29]$ & $0[0.0 ;-0.29,0.29]$ & $1(10 \%)^{a}[0.1 ;-0.26,0.43]$ & $0[0.0 ;-0.29,0.29]$ & $0[0.0 ;-0.29,0.29]$ & 0.833 \\
gastric reflux & $0[0.0 ;-0.29,0.29]$ & $0[0.0 ;-0.29,0.29]$ & $2(20 \%)^{a}[0.2 ;-0.21,0.55]$ & $0[0.0 ;-0.29,0.29]$ & $0[0.0 ;-0.29,0.29]$ & 0.127 \\
\hline
\end{tabular}

Results are given as absolute and relative prevalence values, $\mathrm{n}(\%)$ [estimates; lower and upper confidence limits with $95 \%$ confidence interval] calculated by the Agresti-Caffo add-4 Cl (Agresti \& Caffo, 2000) [38] considering the difference of proportions in each time point versus baseline condition using the multivariate normal distribution (Dunnett) standard normal quantiles. Prevalence was registered when a score of " 5 " or higher arbitrary unit (out of " $10^{\prime \prime}$ ) was informed. P-value calculated from the maximum of test statistics with unpooled variance estimators. Prevalence criterion used was supported by Jeukendrup et al. (2000) [35]. FMP = football match simulation protocol. ${ }^{a}$ concomitance with bloating. ${ }^{b}$ follow-up symptom 
Table 2 Sensorial acceptability of experimental chocolate cow's milk

\begin{tabular}{|c|c|c|c|c|c|c|}
\hline \multirow{2}{*}{$\begin{array}{l}\text { Attribute } \\
\text { acceptance }\end{array}$} & \multirow[t]{2}{*}{ Observed answers } & \multirow{2}{*}{$\begin{array}{l}\text { 1st } \\
\text { aliquot }\end{array}$} & \multirow{2}{*}{$\begin{array}{l}\text { 2nd } \\
\text { aliquot }\end{array}$} & \multirow{2}{*}{$\begin{array}{l}\text { 3rd } \\
\text { aliquot }\end{array}$} & \multicolumn{2}{|c|}{ Aliquot effect and agreement degree } \\
\hline & & & & & $x_{2}^{2}(p$-value $)$ & Kendall's W $(95 \% \mathrm{Cl})$ \\
\hline \multirow[t]{4}{*}{ overall perception } & "I really liked it" & $1(10 \%)$ & $1(10 \%)$ & & $0.800(0.670)$ & $0.57(0.53,1)$ \\
\hline & "I really enjoyed it" & $7(70 \%)$ & $7(70 \%)$ & $8(80 \%)$ & & \\
\hline & "I moderately liked it" & $2(20 \%)$ & $2(20 \%)$ & $1(10 \%)$ & & \\
\hline & "I slightly liked it" & & & $1(10 \%)$ & & \\
\hline \multirow[t]{4}{*}{ appearance } & "I really liked it" & $2(20 \%)$ & $3(30 \%)$ & $2(50 \%)$ & $0.667(0.716)$ & $0.87(0.85,1)$ \\
\hline & "I really enjoyed it" & $6(60 \%)$ & $4(40 \%)$ & $5(50 \%)$ & & \\
\hline & "I moderately liked it" & & & & & \\
\hline & "I slightly liked it" & $2(20 \%)$ & $3(30 \%)$ & $3(30 \%)$ & & \\
\hline \multirow[t]{4}{*}{ consistency } & "I really liked it" & $2(20 \%)$ & $3(30 \%)$ & $3(30 \%)$ & $0.000(1.000)$ & $0.81(0.79,1)$ \\
\hline & "I really enjoyed it" & $6(60 \%)$ & $4(40 \%)$ & $4(40 \%)$ & & \\
\hline & "I moderately liked it" & $2(20 \%)$ & $2(20 \%)$ & $2(20 \%)$ & & \\
\hline & "I slightly liked it" & & $1(10 \%)$ & $1(10 \%)$ & & \\
\hline \multirow[t]{4}{*}{ flavor } & "I really liked it" & $2(50 \%)$ & $1(10 \%)$ & $2(20 \%)$ & $0.737(0.692)$ & $0.63(0.59,1)$ \\
\hline & "I really enjoyed it" & $5(50 \%)$ & $5(50 \%)$ & $4(40 \%)$ & & \\
\hline & "I moderately liked it" & $3(30 \%)$ & $3(30 \%)$ & $3(30 \%)$ & & \\
\hline & "I slightly liked it" & & $1(10 \%)$ & $1(10 \%)$ & & \\
\hline
\end{tabular}

Results are given as absolute and relative value, $\mathrm{n}(\%)$. Total beverage intake $=625 \pm 61 \mathrm{~mL}$. Aliquot intake $=50,30$ and $20 \%$ at first $(0 \mathrm{~min})$, second $(45 \mathrm{~min})$ and third (75 min) post-FMP, respectively. The sensory acceptability criterion was the 9-point hedonic scale proposed by Meilgaard et al. (2006) [36]. $x^{2}{ }_{2}$, chi squared with $\mathrm{df}=2$ and $p$-value were obtained by Friedman rank sum test (used for to verify the time effect). Kendall's W with bootstrapped $95 \% \mathrm{Cl}$ (confidence interval with bootstrap [1000 replicates recalculated from original data]) was used to describe agreement among respondents

demand, as well as to create viable plans for use in daily sport contexts. Thus, the present study formulated a CML prototype in aiming to meet this applicability and technological gap. Furthermore, this ready-to-drink sport-specific drink was evaluated on sensorial acceptability and gastrointestinal complaint scores using football players as potential consumers in an ecological team sport workload and recovery in a real sporting setting. The FMP (adapted $\mathrm{SAFT}^{90}$ ) had similar physiological [39] or mechanical load [29] to football games, reinforcing the validity of the protocol as an instrument for fatigue induction corresponding to an official football match as demonstrated in the external validation study of this protocol [30].

Table 3 Sensory attribute acceptability of experimental chocolate cow milk anchored on a 9-point hedonic scale

\begin{tabular}{ll}
\hline Sensory attributes & mean $\pm \mathbf{s d}(\mathbf{9 5} \% \mathbf{C l})$ \\
\hline Overall perception & $7.8 \pm 0.4(7.5,8.1)$ \\
Appearance & $7.7 \pm 0.9(7.1,8.3)$ \\
Consistency & $7.9 \pm 0.6(7.5,8.4)$ \\
Flavour & $7.7 \pm 0.7(7.3,8.1)$ \\
\hline Results are given as mean \pm sd and $95 \% \mathrm{Cl}$ (confidence interval with bootstrap \\
[1000 replicates recalculated from original data]). Total beverage intake = \\
$625 \pm 61$ mL. The sensory acceptability criterion was the 9-point hedonic scale \\
proposed by Meilgaard et al. (2006) [36]
\end{tabular}

In agreement with our hypotheses, the results demonstrate that the plausible short-term CML benefits do not compromise adherence to a supplementation plan in an immediate post-workout recovery timeline via severe gastrointestinal complaints or for not liking the beverage. Therefore, we believe that the results of the CML are encouraging because it indicates that athletes will not lose their adherence to the proposed supplementation plan. For example, an expressive result for CML was that the majority of athletes answered "I really liked it" or "really enjoyed it", pointing out a higher and consistent timeline response for the product acceptability index $(\sim 88 \%)$ [37]. In addition, this intervention approach with multi-ingredient supply was proposed to match the physical demands which referenced-team sports could create, providing more valuable evidence for athletes to adhere to strategic recovery plan, and how it affects post-match nutrition intervention.

Although preference and gastrointestinal complaints about CML use were not tested against conventional sports drinks or commercial chocolate milk (in order to control confounding variables), and the fact that all athletes had dairy tolerance (motivated to safety), the relatively low prevalence (30\%) of severe bloating symptoms observed 2-h post-intake did not extrapolate the cut-off point of five points from any gastrointestinal discomfort criteria. Thus, the observed scores did not compromise 
the gastrointestinal sensation, being classified as "nonsevere" [35], thus making the CML results encouraging for new and expanded studies.

Leucine-enriched chocolate milk, as present in the CML prototype, is interesting for athletes because they may help preserve their skeletal muscle during various catabolic states, as well as stimulate MPS via insulindependent anabolic stimuli or via $m T O R$ during early exercise recovery [23, 24]. Unfortunately, isolated leucine supplementation could trigger symptoms such as bloating, flatulence, and abdominal pain. Moreover, there is evidence that leucine may have some effects on specific hypothalamic-brainstem circuits which connect amino acid availability and nutrient detection to food intake control [40]. As a result, dietary leucine largely escapes first-pass metabolism, plasma leucine levels are rapidly and markedly increased, and it is likely to represent a physiological signal of hypothalamic amino acid availability [40]. Thus, the combination of chocolate cow milk with one of the most important EAAs may be a valid supplementation strategy to consider. Furthermore, the intake protocol through fractions (aliquots) made the CML intake safe, tolerable, and physiologically interesting for immediate recovery nutritional intervention.

The issues of sensory acceptability and gastrointestinal complaints are important in practice, as athletes cannot stop eating for non-compliance and for reasons due to gastrointestinal discomfort in later stages of recovery. Thus, the bloating gastrointestinal complaint impact of CML was small, considering that other conditions and workload characteristics in team players could aggravate the sensation of gastrointestinal discomfort. In this scope, the present study used a trial which simulates a football match-related fatigue via standardized protocol $[29,30]$. Thus, gastrointestinal or appetite problems could be expected following this exhaustive exercise, as already presented in literature. For example, previous studies have demonstrated slow gastric emptying in football matchplay settings [41] or during a football sport-specific running patterns protocol (Loughborough Intermittent Shuttle Test [LIST]) [42]. Reduced mesenteric blood flow has also previously been shown during intense exercise, especially in hypohydration condition, contributing to the development of gastrointestinal symptoms [43].

The gastrointestinal discomfort was perhaps minimized by the ingestion protocol, although it would be an expected compromise after using milk-based drinks. Cow milk proteins are known for having a low absorption rate due to gastric acid-induced coagulation. Moreover, it can also cause a transient fullness sensation because milk is rich in calcium [44]. Chocolate milk studies in literature have shown controversial results. For example, with a decreased and transiently hunger sensation compared with commercial sport drink (CHO-
Electrolytes) $[10,15]$ or in milk-based beverage use [44]; or with no gastrointestinal complaints for appetite using commercial chocolate milk versus a commercial meal replacement beverage [16].

Due to the new product type in our study, as well as the different dose-response approach, comparisons between previous literature is very difficult, which represents a drawback in the methodology. Studies by Karp et al. [10] and Thomas et al. [15] conducted experiments with athletes being allowed to drink water ad libitum, which may have contributed to triggering the sensation of stomach distension. Thus, the likely transient satiation sensation may be dependent on energy density, composition, and especially intake protocol. Therefore, the gastrointestinal complaint phenomenon could transiently compromise an athlete's gastrointestinal sensation and hunger in post-exercise phase, and CML and its intake protocol seemed to prevent these problems.

No studies were found which used sensory analysis to evaluate any similarity between leucine-supplemented milk and commercial chocolate beverages, for example. Despite this, it is possible to note that the present experimental product acceptance results and its attributes are reasonable and indicative that additional development and studies of CML could be continued. For example, CML showed higher flavour values in comparison with one study evaluating three chocolate drinks which are currently marketed in Brazil, including the market leader, using the same attributes of "overall perception" and "flavour", presenting values at about 6 a.u [45].. However, this study evaluated sensory acceptability at post-exercise moments as done in the present study when factors such as physiological and psychological factors may cause different sensorial evaluations [37]. In addition, our study method used habitual or potential consumers in a real-world context (e.g., football players), dismissing trained tasters. Further studies and evaluations in the scope of food science and technology with physicochemical parameters are also recommended to gain a holistic understanding of drivers for liking the CML product and to continue its development to a larger scale of consumers.

In addition to the promising results of the researched CML intervention, several limitations need to be considered when interpreting its results. First, the sample was limited in size, and the one-group study design lacks a practical or ecological validity control condition. Secondly, discrepancies in composition or energy density, different solubilization rates, and the glycemic index would make the inference of results obscure (i.e., not ideal taste-matched placebo or control) if conventional sports drinks/commercial chocolate milk were used in the study protocol. In addition, the sampled athletes are users of the market leader sports drink in their usual 
routine in training, games, and post-workout. Thus, the authors in this study tried to avoid any previous involvement with brands and commercial products so as to avoid bias, because when consumers know certain product ingredients and expectations or when a pre-existing perception of the ingredient can influence the liking drivers [17]. We also consider that the experimental product in this study is unprecedented, and that comparisons with local chocolate drinks would not provide relevant information. After all, chocolate drinks in local legislation have distinct characteristics of product identity and would be distant from the supplementation recommendations for athletes, because they have a lot of sugar, no enrichment and loose most of the natural benefits of milk through the thermal process (UHT) and sterilization for shelf life.

Regardless of our sample characteristic, or limited hydration protocol conducted with only water before and during exhaustive exercise, it could be plausible that CML prototype and its intake protocol proved to be an attractive post-match nutrition strategy for football players. CML energy intake $(\sim 700 \mathrm{kcal})$ enabled meeting approximately $50 \%$ of energy requirements in an official football match in elite athletes [46], and may help to increase adherence to athletes' nutritional plans according to the practicality for logistics in a competitive scenario. Future studies can investigate the effectiveness of milkbased beverage intake, such as CML, on metabolism, immune state and redox, postprandial fullness sensation, rehydration, performance, and appetite effect on young athletes to professional-standard players.

\section{Conclusion}

These preliminary findings suggest that CML may be an important nutritional intervention for use in post-highdemand exercise while maintaining palatability and with no severe gastrointestinal complaints. The lactose-free CML and leucine-enriched formulation, as well as its intake protocol, could be strategies which could expand the user public as it is a safer and team-sports demandsdriven approach of chocolate milk. Moreover, it can help in balancing the nutritional composition and postworkout drink effectiveness. Furthermore, some multiingredient characteristics, such as CML along with the presence of other nutrients such as antioxidants, amino acids, vitamins, and minerals, may be advantageous over conventional sports drinks or isolated micronutrient supplements. Additional studies are recommended to examine the proof-of-concept solution and the potential advantages of this new sport-specific drink and its mechanisms involved in recovery from fatigue from teamsports demands in elite players, including for long-term use.

\begin{abstract}
Abbreviations
$\% H R_{\text {peak: }}$ Percentage of heart rate peak; a.u.: arbitrary unit; BW: Body weight; CHO: Carbohydrate; Cl: Confidence intervals; CML: Chocolate cow milk enriched with L-leucine; FMP: Football match protocol (adapted version of SAFT $^{90}$ : soccer-specific aerobic field test); MPS: Muscle protein synthesis; METs: Metabolic equivalents; mTOR: mammalian target of rapamycin; PRO: Protein; RH: Relative humidity; SD: Standard deviation; UHT: Ultra-high temperature; USG: Urine specific gravity
\end{abstract}

\section{Acknowledgments}

The authors would like to thank the individuals who volunteered to participate in the study.

\section{Authors' contributions}

Conceptualization, CDS; methodology, CDS, DRO and ESG; validation, CDS and ESG; formal analysis, CDS, ITP and CHF; investigation, CDS and ITP; resources, CDS; data curation, CDS and DRO; writing_-original draft preparation, CDS; writing-review and editing, CDS, DRO, CHF, and ESG; visualization, CDS and DRO; supervision, CDS, ITP and ESG; project administration CDS and ESG. All authors have read and agreed to the published version of the manuscript.

\section{Funding}

The authors declare that they had not received any specific grant for this research from any funding agency in the public, commercial or non-profit sectors. This study was part of a doctoral degree and the submitted research article has been privately funded.

\section{Availability of data and materials}

Please contact author for data requests.

\section{Ethics approval and consent to participate}

This study was approved by the Institutional Review Board at the Federal University of Minas Gerais, Brazil (CAAE: 58632153514.5.0000.5149). All participantsprovided written consent.

\section{Consent for publication}

As part of the written informed consent procedure, all participants were duly made aware (as part of both the study briefing and information sheet) that the study results may be published. As such, consent for publication was included as part of this process.

\section{Competing interests}

The authors declare that they have no competing interests. The results of the present study do not constitute endorsement by the authors or J Int Soc Sports Nutr.

\section{Author details}

${ }^{1}$ Department of Physical Education, Institute of Life Sciences, Federal University of Juiz de Fora, Campus: Governador Valadares, Rua Manoel Byrro, 241 - Vila Bretas, Governador Valadares, MG 35010-260, Brazil. ${ }^{2}$ School of Physical Education, Physiotherapy and Occupational Therapy, Federal University of Minas Gerais, Belo Horizonte, MG, Brazil. ${ }^{3}$ Department of Basic Life Sciences Institute of Life Sciences, Federal University of Juiz de Fora, Campus Governador Valadares, MG, Governador Valadares, Brazil. ${ }^{4}$ Department of Food Engineering, Center of Exact Sciences, Federal University of Viçosa, Viçosa, MG, Brazil. ${ }^{5}$ Pharmaceutical Department, Faculty of Pharmacy, Federal University of Juiz de Fora, Campus Juiz de Fora, MG, Brazil. ${ }^{6}$ Pharmaceutical Department, Institute of Life Sciences, Federal University of Juiz de Fora, Campus Governador Valadares, MG, Brazil. ${ }^{7}$ Department of Physical Education, Federal University of Maranhão, São Luís, Maranhão, Brazil.

Received: 6 April 2020 Accepted: 26 December 2020

Published online: 10 February 2021

\section{References}

1. Beelen M, Burke LM, Gibala MJ, van Loon LUC. Nutritional strategies to promote postexercise recovery. Int J Sport Nutr Exerc Metab. 2010;20(6): 515-32. 
2. Kerksick CM, Arent S, Schoenfeld BJ, Stout JR, Campbell B, Wilborn CD, et al. International Society of Sports Nutrition Position Stand: nutrient timing. J Int Soc Sports Nutr. 2017;14:33.

3. Alghannam AF. Carbohydrate-protein ingestion improves subsequent running capacity towards the end of a football-specific intermittent exercise. Appl Physiol Nutr Metab. 2011;36(5):748-57.

4. Highton J, Twist C, Lamb K, Nicholas C. Carbohydrate-protein coingestion improves multiple-sprint running performance. J Sports Sci. 2013;31(4):361-9.

5. I Iy JL, Ding Z, Hwang H, Cialdella-Kam LC, Morrison PJ. Post exercise carbohydrateprotein supplementation: phosphorylation of muscle proteins involved in glycogen synthesis and protein translation. Amino Acids. 2008;35(1):89-97.

6. Wang W, Ding Z, Solares GJ, Choi S, Wang B, Yoon A, et al. Co-ingestion of carbohydrate and whey protein increases fasted rates of muscle protein synthesis immediately after resistance exercise in rats. PLoS One. 2017;12(3): e0173809.

7. Hara D, Morrison PJ, Ding Z, Ivy JL. Effect of carbohydrate-protein supplementation postexercise on rat muscle glycogen synthesis and phosphorylation of proteins controlling glucose storage. Metabolism. 2011; 60(10):1406-15.

8. Morifuji M, Kanda A, Koga J, Kawanaka K, Higuchi M. Post-exercise carbohydrate plus whey protein hydrolysates supplementation increases skeletal muscle glycogen level in rats. Amino Acids. 2010;38(4):1109-15.

9. Ferguson-Stegall L, McCleave EL, Ding Z, Doerner PG, Wang B, Liao Y, et al. Postexercise carbohydrate-protein supplementation improves subsequent exercise performance and intracellular signaling for protein synthesis. J Strength Cond Res. 2011;25(5):1210-24.

10. Karp JR, Johnston JD, Tecklenburg S, Mickleborough TD, Fly AD, Stager JM. Chocolate milk as a post-exercise recovery aid. Int J Sport Nutr Exerc Metab. 2006;16(1):78-91.

11. Spaccarotella KJ, Andzel WD. The effects of low fat chocolate milk on postexercise recovery in collegiate athletes. J Strength Cond Res. 2011; 25(12):3456-60.

12. Born KA, Dooley EE, Cheshire PA, McGill LE, Cosgrove JM, Ivy JL, et al. Chocolate milk versus carbohydrate supplements in adolescent athletes: a field based study. J Int Soc Sports Nutr. 2019;16(1):6.

13. Burke LM. Nutrition for post-exercise recovery. Aust J Sci Med Sport. 1997;29(1):3-10.

14. Hazell TJ, Islam H, Townsend LK, Schmale MS, Copeland JL. Effects of exercise intensity on plasma concentrations of appetite-regulating hormones: Potential mechanisms. Appetite. 2016;98:80-8.

15. Thomas K, Morris P, Stevenson E. Improved endurance capacity following chocolate milk consumption compared with 2 commercially available sport drinks. Appl Physiol Nutr Metab. 2009;34(1):78-82.

16. Pritchett K, Bishop P, Pritchett R, Green M, Katica C. Acute effects of chocolate milk and a commercial recovery beverage on postexercise recovery indices and endurance cycling performance. Appl Physiol Nutr Metab. 2009;34(6):1017-22.

17. Li XE, Jervis SM, Drake MA. Examining extrinsic factors that influence product acceptance: a review. J Food Sci. 2015;80(5):R901-9.

18. Brasil. Ministério da Agricultura, Pecuária e Abastecimento. In: Instrução Normativa $N^{\circ} 16$, de 23 de agosto de 2005. Regulamento técnico de identidade e qualidade de bebidas lácteas. Diário Oficial da República Federativa do Brasil; 2005.

19. Brasil. Agência Nacional de Vigilância Sanitária-Anvisa. Resolução n. 12, de 2 de janeiro de 2001. Regulamento Técnico sobre padrões microbiológicos para alimentos. Diário Oficial da República Federativa do Brasil. 2001: (7):45-53.

20. Jäger R, Kerksick CM, Campbell BI, Cribb PJ, Wells SD, Skwiat TM, et al. International Society of Sports Nutrition Position Stand: protein and exercise. Int Soc Sports Nutr. 2017;14:20.

21. Jentjens $R L$, van Loon $L$, Mann $C H$, Wagenmakers $A J$, Jeukendrup $A E$. Addition of protein and amino acids to carbohydrates does not enhance postexercise muscle glycogen synthesis. J Appl Physiol. 2001;91(2):839-46.

22. Kaastra B, Manders RJF, Van Breda E, Kies A, Jeukendrup AE, Keizer HA, et al. Effects of increasing insulin secretion on acute postexercise blood glucose disposal. Med Sci Sports Exerc. 2006:38(2):268-75.

23. Koopman R, Wagenmakers AJM, Manders RJF, Zorenc AHG, Senden JMG Gorselink $M$, et al. Combined ingestion of protein and free leucine with carbohydrate increases postexercise muscle protein synthesis in vivo in male subjects. Am J Physiol Endocrinol Metab. 2005;288(4):E645-53.

24. Rowlands DS, Nelson AR, Phillips SM, Faulkner JA, Clarke J, Burd NA, et al. Protein-leucine fed dose effects on muscle protein synthesis after endurance exercise. Med Sci Sports Exerc. 2015;47(3):547-55.
25. Manders RJ, Koopman R, Sluijsmans WE, van den Berg R, Verbeek K, Saris $\mathrm{WH}$, et al. Co-ingestion of a protein hydrolysate with or without additional leucine effectively reduces postprandial blood glucose excursions in Type 2 diabetic men. J Nutr. 2006;136(5):1294-9.

26. Areta $\mathrm{L}$, Burke LM, Ross ML, Camera DM, West DWD, Broad EM et al. Timing and distribution of protein ingestion during prolonged recovery from resistance exercise alters myofibrillar protein synthesis. J Physiol 2013; 591(Pt 9):2319-2331.

27. Elango R, Chapman K, Rafii M, Ball RO, Pencharz PB. Determination of the tolerable upper intake level of leucine in acute dietary studies in young men. Am J Clin Nutr. 2012;96(4):759-67.

28. Pencharz PB, Elango R, Ball RO. Determination of the tolerable upper intake level of leucine in adult men. J Nutr. 2012;142(12):2220S-4S.

29. da Silva CD, Machado G, Fernandes AA, Teoldo I, Pimenta EM, Marins JCB, et al. Muscle damage-based recovery strategies can be supported by predictive capacity of specific Global Positioning System Accelerometry parameters immediately a post-soccer match-Load. J Strength Cond Res. 2018; in press.

30. Silva CD, Lovell R. External validity of T-SAFT ${ }^{90}$ : a soccer-simulation including technical and jumping activities. Int J Sports Physiol Perform. 2020;18:1-7.

31. Monteiro MC, Vannucchi H, Dutra de Oliveira JE. Use of the demonstrative method for the quantitative evaluation of food intake. Arch Latinoam Nutr. 1986;36(2):260-7.

32. Volterman KA, Obeid J, Wilk B, Timmons BW. Effect of milk consumption on rehydration in youth following exercise in the heat. Appl Physiol Nutr Metab. 2014;39(11):1257-64.

33. Casa DJ, Armstrong LE, Hillman SK, Montain SJ, Reiff RV, Rich BS, et al. National athletic trainers' association position statement: Fluid replacement for athletes. J Athl Train. 2000;35(2):212-24.

34. Murray R, Eddy DE, Murray TW, Seifert JG, Paul GL, Halaby GA. The effect of fluid and carbohydrate feedings during intermittent cycling exercise. Med Sci Sports Exerc. 1987;19(6):597-604.

35. Jeukendrup AE, Vet-Joop K, Sturk A, Stegen JH, Senden J, Saris WH, et al. Relationship between gastro-intestinal complaints and endotoxaemia, cytokine release and the acute-phase reaction during and after a longdistance triathlon in highly trained men. Clin Sci. 2000;98(1):47-55.

36. Meilgaard M, Civille G, Carr B. Sensory Evaluation Techniques. 4th ed. Boca Raton: CRC Press/Taylor \& Francis; 2006.

37. Dutcosky SD. Análise sensorial de alimentos. Curitiba: Champagnat Pucpress; 2013.

38. Agresti A, Caffo B. Simple and effective confidence intervals for proportions and differences of proportions result from adding two successes and two failures. Am Stat. 2000;54(4):280-8.

39. Dellal A, Da Silva CD, Hill-Haas S, Wong DP, Natali AJ, De Lima JRP, et al. Heart-rate monitoring in soccer: interest and limits during competitive match-play and training - Practical application. J Strength Cond Res. 2012; 26(10):2890-906.

40. Blouet C, Jo Y, Li X, Schwartz GJ. Mediobasal hypothalamic leucine sensing regulates food intake through activation of a hypothalamus-brainstem circuit. J Neurosci. 2009:29(26):8302-11.

41. Leiper JB, Prentice AS, Wrightson C, Maughan RJ. Gastric emptying of a carbohydrate-electrolyte drink during a soccer match. Med Sci Sports Exerc. 2001;33(11):1932-8.

42. Leiper JB, Nicholas CW, Ali A, Williams C, Maughan RJ. The effect of intermittent high-intensity running on gastric emptying of fluids in man. Med Sci Sports Exerc. 2005;37(2):240-7.

43. de Oliveira EP, Burini RC, Jeukendrup A. Gastrointestinal complaints during exercise: prevalence, etiology, and nutritional recommendations. Sports Med. 2014:44(Suppl 1):S79-85.

44. Gonzalez JT, Rumbold PLS, Stevenson EJ. Appetite sensations and substrate metabolism at rest, during exercise, and recovery: impact of a high-calcium meal. Appl Physiol Nutr Metab. 2013;38(12):1260-7.

45. Pflanzer SB, Cruz AGD, Hatanaka CL, Mamede PL, Cadena R, Faria JAF, et al. Perfil sensorial e aceitação de bebida láctea achocolatada. Food Sci Technol. 2010:30:391-8.

46. Bangsbo J. Energy demands in competitive soccer. J Sports Sci. 1994;12:S5-12.

\section{Publisher's Note}

Springer Nature remains neutral with regard to jurisdictional claims in published maps and institutional affiliations. 